\title{
Reply from Pakzad
}

\author{
Hamid Reza Pakzad
}

Received: 18 January 2010 / Accepted: 18 January 2010 / Published online: 12 February 2010

(C) Springer Science+Business Media B.V. 2010

\section{Dear Editor,}

The main point in Verheest \& Pillay's "Comment on Dust acoustic solitary waves in dusty plasma with nonthermal ions", against my article which appeared in Astrophys. Space Sci. 324, 41-45 (2009) is that the authors had published a more complete article than the above manuscript about a year and a half earlier. They conclude that Pakzad did not survey the mainstream literature before publishing the manuscript. Here are some suggestions which may help authors to revise their opinion.

A short examination of the previous articles by Pakzad reveals that the above manuscript is a natural expansion of his previous works. The paper mainly deals with the conditions for the existence of solitary waves in a medium containing nonthermal electrons. As stated in this manuscript, this point is widely applicable-independent of the double layer situation which has been investigated in the nice work of Verheest and collaborators. In fact Pakzad's work is both parallel to (and also independent of) the very complete work presented by Verheest et al. The referee for our paper considered this point in his/her final decision. To find both parallel and independent works is not surprising news in view of the recent situation of knowledge and specially in plasma physics. One can find that all the articles cited below have investigated the same problems as Verheest both before and after their work.

This letter to the editor is related to the other letter available at 10.1007/s10509-010-0285-2.

\section{H.R. Pakzad $(\bowtie)$}

Department of Physics, Islamic Azad University,

Bojnourd Branch, Iran

e-mail: pakzad@bojnourdiau.ac.ir
Another point worth noting is what is meant by "the mainstream literature". This is very dependent upon the available sources an author has at hand. This is not the same in places all around the world. There are very great differences between the scientific resources and funding available in the great research groups in developed countries and those in a small town of a developing country.

In conclusion I affirm that my work provides another outlook to the same problem fully investigated by Verheest et al. and may give different inspiration to the reader.

\section{References}

Aoutou, K.: Electrostatic solitary structures in dusty plasmas with nonthermal and superthermal electrons. Phys. Plasmas 15, 103702 (2008)

Ali-Fedela, D., et al.: Arbitrary amplitude ion-acoustic waves in a plasma with nonthermal electrons. Adv. Space Res. (2009, in press)

Djeblia, M., Marif, H.: Large amplitude double layers in a positively charged dusty plasma with nonthermal electrons. Phys. Plasmas 16, 063708 (2009)

El-Taibany, W.F., Kourakis, L.: Modulational instability of dust acoustic waves in dusty plasmas: Modulation obliqueness, background ion nonthermality, and dust charging effects. Phys. Plasmas 13, 062302 (2006)

El-Taibany, W.F., Wadati, M.: Sagdeev potential analysis for positively charged dust grains in nonthermal dusty plasma near Mars. Phys. Plasmas 14, 103703 (2007)

El-Taibany, W.F., Wadati, M., Sabry, R.: Nonlinear dust acoustic waves in a nonuniform magnetized complex plasma with nonthermal ions and dust charge variation. Phys. Plasmas 14, 032304 (2007)

Gill, T.S., et al.: Effect of nonthermal ion distribution and dust temperature on nonlinear dust acoustic solitary waves. PRAMANAJ. Phys. 55, 855-859 (2000)

Mandal, G., et al.: Large amplitude double layers in a four component dusty plasma with non-thermal ions. Indian J. Phys. 3, 365-374 (2009)

Misra, G., et al.: Modulational instability of dust acoustic waves in a dusty plasma with nonthermal electrons and ions. Eur. Phys. J. D 39, 47-57 (2006) 